\title{
Focal Stimulation of the Posterior Parietal Cortex Increases the Excitability of the Ipsilateral Motor Cortex
}

\author{
Giacomo Koch, ${ }^{1,2,3}$ Miguel Fernandez Del Olmo, ${ }^{1,4}$ Binith Cheeran, ${ }^{1}$ Diane Ruge, ${ }^{1}$ Sven Schippling, ${ }^{1,5}$ \\ Carlo Caltagirone, ${ }^{2,3}$ and John C. Rothwell ${ }^{1}$ \\ ${ }^{1}$ Sobell Department of Motor Neuroscience and Movement Disorders, Institute of Neurology, University College London, Queen Square, London WC1N \\ 3BG, United Kingdom, ${ }^{2}$ Laboratorio di Neurologia Clinica e Comportamentale, Fondazione Santa Lucia, 00179 Rome, Italy, ${ }^{3}$ Clinica Neurologica, \\ Dipartimento di Neuroscienze, Università di Roma Tor Vergata, 00133 Rome, Italy, ${ }^{4}$ Institute of Physical Education and Sport, La Coruña, Spain, and \\ ${ }^{5}$ Department of Neurology, University Medical Centre Hamburg-Eppendorf, Germany
}

Paired-pulse transcranial magnetic stimulation (TMS) has been applied as a probe to test functional connectivity within distinct cortical areas of the human motor system. Here, we tested the interaction between the posterior parietal cortex (PPC) and ipsilateral motor cortex (M1). A conditioning TMS pulse over the right PPC potentiates motor evoked-potentials evoked by a test TMS pulse over the ipsilateral motor cortex, with a time course characterized by two phases: an early peak at $4 \mathrm{~ms}$ interstimulus interval (ISI) and a late peak at $15 \mathrm{~ms}$ ISI. Activation of this facilitatory pathway depends on the intensity of stimulation, because the effects are induced with a conditioning stimulus of $90 \%$ resting motor threshold but not at lower or higher intensities. Similar results were obtained testing the ipsilateral interaction in the left hemisphere with a slightly different time course. In control experiments, we found that activation of this facilitatory pathway depends on the direction of induced current in the brain and is specific for stimulation of the caudal part of the inferior parietal sulcus (cIPS) site, because it is not observed for stimulation of adjacent scalp sites. Finally, we found that by using poststimulus time histogram analysis of single motor unit firing, the PPC conditioning increases the excitability of ipsilateral M1, enhancing the relative amount of late I wave input recruited by the test stimulus over M1, suggesting that such interaction is mediated by specific interneurons in the motor cortex. The described facilitatory connections between cIPS and M1 may be important in a variety of motor tasks and neuropsychiatric disorders.

Key words: connectivity; facilitation; motor control; motor cortex; motor-evoked potential; parietal

\section{Introduction}

The pioneering studies of Meynert (1865) and Dejerine (1895) revealed that the posterior parietal cortex (PPC) is strongly interconnected with motor, premotor, and more frontal areas of the cortex through distinct white matter tracts that form the superior longitudinal fasciculus (SLF). These corticocortical connections are thought to transfer crucial information relevant for planning movements in space and to integrate visuomotor transformations (Mountcastle et al., 1975; Cohen and Andersen, 2002; Tanne-Gariepy et al., 2002; Croxson et al., 2005; Makris et al., 2005; Rozzi et al., 2006; Rushworth et al., 2006). Previous combined transcranial magnetic stimulation (TMS)/positron emission tomography, TMS/functional magnetic resonance imaging (MRI), and paired TMS investigations have shown that TMS not only changes neural activity at the site of stimulation but also affects interconnected cortical and subcortical areas (Ferbert et al., 1992; Ugawa et al., 1995; Paus et al., 1997; Lee et al., 2003;

Received Feb. 11, 2007; revised April 27, 2007; accepted May 1, 2007.

M.F.D.O. was supported by a grant from Xunta de Galicia (PGIDIT06PXIB160333PR), Spain.

Correspondence should be addressed to Prof. John C. Rothwell, Sobell Department of Motor Neuroscience and Movement Disorders, Institute of Neurology, University College London, Queen Square, London WC1N 3BG, United Kingdom. E-mail: J.Rothwell@ion.ucl.ac.uk.

DOI:10.1523/JNEUROSCI.0598-07.2007

Copyright $\odot 2007$ Society for Neuroscience $\quad$ 0270-6474/07/276815-08\$15.00/0
Bestmann et al., 2004, 2005). Paired-pulse TMS provides a unique opportunity in humans to probe inputs to the primary motor cortex from other areas of the motor system. A conditioning stimulus (CS) is first used to activate putative pathways to the motor cortex from the site of stimulation, whereas a second test stimulus (TS) delivered over the primary motor cortex a few milliseconds later probes any changes in excitability that are produced by the input. Depending on the intensity of the conditioning stimulus and the interstimulus interval, both facilitation and inhibition may be detected in the primary motor cortex (M1), ipsilateral or contralateral to the site of conditioning. Previous studies have been conducted with the CS delivered over the contralateral M1 (Ferbert et al., 1992), the cerebellum (Ugawa et al. 1995), and the premotor cortex (Civardi et al., 2001; Mochizucki et al., 2004; Baumer et al., 2006; Koch et al., 2006, 2007), confirming the existence of these pathways in humans.

However, the advantage of probing these pathways with TMS methods is that the response to a TMS conditioning pulse depends on the excitability of the pathway at the time the stimulus is applied. Thus, changes in the effectiveness of the conditioning pulse give an indication of how the excitability of the connection changes over time. Such an approach has been used to show how transcallosal inhibition from the M1 of one hemisphere is removed before the onset of movements made by the opposite 
hemisphere both in healthy subjects and in patients after stroke (Murase et al., 2004). Recently, we used a similar approach to show how dorsal premotor (PMd)-M1 connectivity changed critically during preparation of movement (Koch et al., 2006). Given the importance of input from posterior parietal regions in visuomotor planning, we tested in the present study whether stimulating the PPC had any effects on the excitability of the ipsilateral primary motor cortex. If so, then it may prove possible in future studies to use this method to probe the time course of PPC-M1 connectivity during movement and to test how this is impaired in different neurological disorders such as neglect (Oliveri et al., 1999, 2000).

\section{Materials and Methods}

Subjects. Twenty healthy volunteers (11 men and 9 women, $21-50$ years old) participated in this study. All subjects were right handed based on the Edinburgh Handedness Inventory. Written informed consent was obtained from all subjects. The experimental procedures used here were approved by the local Ethics Committee and were performed in accordance with the Declaration of Helsinki.

Experimental procedures. Electromyographic (EMG) recordings were made from the first dorsal interosseous (FDI) muscles using $9 \mathrm{~mm}$ diameter, $\mathrm{Ag}-\mathrm{AgCl}$ surface-cup electrodes. The active electrode was placed over the muscle belly and the reference electrode over the metacarpophalangeal joint of the index finger. Responses were amplified with a Digitimer D360 amplifier (Digitimer, Welwyn Garden City, UK) through filters set at $20 \mathrm{~Hz}$ and $2 \mathrm{kHz}$ with a sampling rate of $5 \mathrm{kHz}$, then recorded by a computer using SIGNAL software (Cambridge Electronic Devices, Cambridge, UK). We used a paired-pulse stimulation technique with two high-power Magstim 200 machines (Magstim, Whitland, UK). The magnetic stimulus had a nearly monophasic pulse configuration with a rise time of $\sim 100 \mu \mathrm{s}$, decaying back to zero over $\sim 0.8 \mathrm{~ms}$.

Experiment 1: PPC-M1 interactions in the right hemisphere. Ten subjects participated in this experiment. We used a paired-pulse stimulation technique with two high-power Magstim 200 machines (Magstim). First, the intensity of TS was adjusted to evoke a motor-evoked potential (MEP) of $\sim 1 \mathrm{mV}$ peak to peak in the relaxed left FDI. The hand motor area of the right M1 was defined as the point where stimulation evoked the largest MEP from the contralateral FDI muscle. The test stimulator was connected to a small custom-made figure-of-eight-shaped coil (external diameter, $50 \mathrm{~mm}$ ). To stimulate $\mathrm{M} 1$, the coil was always placed tangentially to the scalp at a $45^{\circ}$ angle to the midline to induce a posterior-anterior (PA) current flow across the central sulcus.

The conditioning stimulator was connected to a larger figure-of-eightshaped coil, $70 \mathrm{~mm}$ in external diameter The coil position for the right PPC TMS was then defined relative to the P4 position of the 10-20 EEG system. According to previous investigations adopting threedimensional (3D) MRI reconstruction, this site is situated in the inferior parietal lobule (IPL) close to the posterior part of the adjoining intraparietal sulcus (pIPS) (Herwig et al., 2003; Rushworth and Taylor, 2006). The center of the coil was positioned over P4 tangentially to the skull with the handle pointing downward and slightly medial $\left(10^{\circ}\right)$ to induce a posterior-anterior-directed current in the underlying cortical tissue. This orientation was chosen because of space considerations; it was the only approach in which the two figure-of-eight coils did not overlap. In three subjects, MRI-guided frameless stereotaxy (Brainsight Frameless; Rogue Research, Montreal, Quebec, Canada) was used to verify the position of the coil with respect to the underlying cortex (see Fig. 1 A.). The intensity of the CS stimulus was adjusted to be either suprathreshold [130 and $110 \%$ resting motor threshold (RMT)] or subthreshold (90 and $70 \%$ RMT). For CS, RMT was tested with the larger figure-of-eight coil over the ipsilateral M1 using a PA orientation. It is in fact known that the coil dimension significantly affect the intensity and 3D distribution of the induced currents flowing within the brain; the small and the large figureof-eight coils do not have the same RMT and, therefore, $90-130 \%$ of values were relative to the RMT of the larger coil. We defined RMT as the lowest intensity that evoked five small responses $(\sim 50 \mu \mathrm{V})$ in the contralateral FDI muscle in a series of 10 stimuli when the subject kept the
FDI muscles relaxed in both hands according to international standards (Rossini et al., 1994).

Interstimulus intervals (ISIs) between CS and TS were 2, 4, 6, 8, 10, 15, and $20 \mathrm{~ms}$. In each block, we varied the intensity of the CS, and the order of presentation of blocks varied pseudorandomly across subjects. Each block consisted of ninety trials. Eight conditions were randomly intermingled: TS alone (MEP) and CS1 plus TS (conditioned MEP for each seven different ISIs). Twenty responses were collected for the test stimulus alone and 10 responses for conditioned MEPs at each ISI. There was a $5 \mathrm{~s}( \pm 10 \%)$ intertrial interval. In four of the same subjects plus two others, we also investigated ISIs of $-1,-2,-3$, and $4 \mathrm{~ms}$ in a separate block of trials using a CS of $90 \%$ RMT. Measurements were made on each individual trial and the mean peak-to-peak amplitude of the conditioned MEP was expressed as a percentage of the mean peak-to-peak amplitude of the unconditioned test pulse (see Fig. $1 A$ ).

Experiment 2: effects of reversing the direction of current in the conditioning coil. In this control experiment, we varied the direction of current in the conditioning coil. The experiment was performed in six subjects (four of whom participated in experiment 1). CS and TS were applied as in experiment 1 . On the basis of the findings in experiment 1 , the intensity of the CS stimulus was set at $90 \%$ RMT. ISIs between CS and TS were $2,4,6,8$, and $10 \mathrm{~ms}$. In each block, we varied the current direction of the CS through a dedicated device (Magstim). The order of presentation of blocks varied pseudorandomly across subjects. Each block consisted of seventy trials. Six conditions were randomly intermingled: TS alone (MEP) and CS1 plus TS (conditioned MEP for each five different ISIs). Twenty responses were collected for the test stimulus alone and 10 responses for conditioned MEPs at each ISI. There was a $5 \mathrm{~s}( \pm 10 \%)$ intertrial interval. Measurements were made on each individual trial and the mean peak-to-peak amplitude of the conditioned MEP was expressed as a percentage of the mean peak-to-peak amplitude of the unconditioned test pulse.

Experiment 3: effects of changing the location of the conditioning stimulus. In these experiments, we investigated the effects of applying the CS over two scalp sites close to P4. In six subjects (all of whom had taken part in experiment 1), a CS was applied either to $\mathrm{P} 4$ or to a position $2 \mathrm{~cm}$ medial or $2 \mathrm{~cm}$ lateral. The intensity of the CS was adjusted to be $90 \%$ RMT. ISIs between CS and TS were 2, 4, 6, 8, and 10 ms. Each block for each scalp site consisted of seventy trials. Five conditions were randomly intermingled: TS alone (MEP) and CS1 plus TS (conditioned MEP for each five different ISIs). Twenty responses were collected for the test stimulus alone and 10 responses for conditioned MEPs at each ISI. The order of presentation of blocks varied pseudorandomly across subjects. Measurements were made on each individual trial and the mean peakto-peak amplitude of the conditioned MEP was also expressed as a percentage of the mean peak-to-peak amplitude size of the unconditioned test pulse.

In an additional experiment performed in four subjects in which MRI had been previously obtained, three cortical sites along the IPS of the right hemisphere were chosen for application of the conditioning stimulus: (1) the most anterior region of the IPS (aIPS), located between the inferior postcentral sulcus and the most anterior extent of the IPS, (2) a region in the middle of the sulcus (mIPS), and (3) a region in the most caudal part of the IPS (cIPS) (see Fig. 3A).

A high resolution three-dimensional volumetric structural magnetic resonance image (MRI) was obtained. The cortical surface was displayed as a three-dimensional representation using Brainsight Frameless Stereotaxic software (Rogue Research). Each of the three targeted cortical stimulation sites on the individual three-dimensional MRI was demarcated using a pointer tool (Brainsight Frameless P-697) and the same software. The corresponding scalp position was marked using a pen. During the procedure, the position of the pointer and the subject's head were monitored using a Polaris Optical Tracking system (Northern Digital, Waterloo, Ontario, Canada). Positional data for head and pointer were registered in real time to a common frame of reference and were superimposed onto the reconstructed three- dimensional image of each subject using the same software.

We again used a paired-pulse stimulation with two high-power Magstim 200 stimulators (Magstim). For each of the three targeted stimula- 
tion sites, the CS was applied over the corresponding scalp position using a small custom-made figure-of-eight shaped coil (external diameter, 50 $\mathrm{mm}$ ). The coil was kept tangentially to the skull with the handle pointing downward and slightly medially $\left(10^{\circ}\right)$. The TS was applied over the motor area of M1 where stimulation evoked the largest MEP from the contralateral FDI muscle using a circular coil (external diameter, $100 \mathrm{~mm}$ ) with a clockwise current flow in the coil. This coil combination was chosen to achieve minimal spatial interference of the two coils. Therefore, in this experiment, the coil for conditioning PPC was smaller (50 $\mathrm{mm}$ ) then the larger one used in the previous experiments. However, the same stimulators were used in all experiments. The intensity of the TS was adjusted to evoke a MEP of $\sim 1 \mathrm{mV}$ peak to peak in the relaxed muscle. The intensity of the CS was kept at an intensity of $90 \%$ RMT. The ISI between CS and TS was 4 ms (12 stimuli per condition, randomly intermixed). The stimulation site of the CS was varied randomly between subjects. There was a $5 \mathrm{~s}( \pm 10 \%)$ intertrial interval. Measurements were made on each individual trial and the mean peak-to-peak amplitude of the conditioned MEP was expressed as a percentage of the mean peakto-peak amplitude of the unconditioned test pulse.

Experiment 4: effects of stimulating the right PPC on the pattern of single motor unit discharge evoked by a test stimulus over M1. In this experiment, we studied the effect of PPC conditioning on the discharge of single motor units (SMU) evoked by TMS over M1. SMU were recorded from the left FDI with a concentric needle electrode (Medelec, disposable type DML25). Signals were amplified through filters set at $100 \mathrm{~Hz}$ and $3 \mathrm{kHz}$. The subjects were instructed to fire the unit voluntarily at $\sim 10 \mathrm{~Hz}$ with the aid of audiovisual feedback, and TMS pulses were then applied to M1 every 3-4 s. The effect on unit firing was assessed by constructing a poststimulus time histogram (PSTH) of unit discharge with a bin width of $0.5 \mathrm{~ms}$. The threshold of the first-recruited peak in the PSTH was first determined by changing the intensity of stimulation in steps of $2 \%$ of the maximum stimulator output. The threshold was defined as the lowest intensity that evoked a small peak in the PSTH. These thresholds were almost the same (difference $<5 \%$ of maximum stimulator output) as those for surface EMG responses during a slight contraction. We studied only the first recruited SMU, so that we were able to isolate their discharge easily. Therefore, we did not select SMU on the basis of different sizes with different thresholds of recruitment according to the Hennemann's law (Rossini et al., 1995). The intensity of TS was then adjusted to produce $20-30 \%$ firing probability, which was $10-15 \%$ above the threshold. Two kinds of PSTHs were recorded simultaneously. One was a PSTH evoked by TS given alone (control PSTH); the other was a PSTH when both stimuli (CS plus TS) were given (conditioned PSTH). Control and conditioned trials were intermixed randomly by the computer until 100 trials had been collected for each condition (Hanajima et al., 2002), and a PSTH was then constructed for each condition. The orientation of the CS and TS were as in experiment 1; the CS stimulus intensity was set at $90 \%$ RMT with an ISI of $4 \mathrm{~ms}$. Ten motor units from seven subjects were studied. To compare PSTHs across subjects, we divided the period of increased firing into three parts (every $1.5 \mathrm{~ms}$ ) in each subject, starting from the first peak in the PSTH consistent with the individual surface MEP latency.

Experiment 5: effects of stimulating right PPC on the H reflex evoked by median nerve stimulation. Five subjects participated in this control experiment. We compared the effect of PPC conditioning on MEPs and spinal $\mathrm{H}$ reflexes in the flexor carpi radialis (FCR) muscle. The excitability of the monosynaptic H-reflex in the left FCR motoneurons was tested with standard technique (Hortobágyi et al., 2003). Single electrical stimuli were delivered with saline-soaked gauze-covered button electrodes, the cathode $5 \mathrm{~cm}$ proximal to the anode, in the cubital space (duration, $1 \mathrm{~ms}$; DS7; Digitimer) with the subject at rest. First, we determined the appropriate stimulating electrode location and identified the H-reflex in the FCR based on its latency and recruitment curve. The median nerve stimulation intensity was set to produce an H-reflex that corresponded to an amplitude of $\sim 0.5-1 \mathrm{mV}$. The intensity of the PPC CS was adjusted to be $90 \%$ RMT. The intensity of motor cortex stimulation was set to produce a MEP recorded from the left FCR that corresponded to an amplitude of $\sim 0.5-1 \mathrm{mV}$. There were four conditions: (1) MEPs recorded after stimulation of the hot spot of the FCR $(t=0 \mathrm{~ms})$; (2) MEP conditioned by
PPC CS using an ISI between CS and TS of $4 \mathrm{~ms}(t=-4 \mathrm{~ms}$ ); (3) FCR $\mathrm{H}$-reflex with the time of the median nerve stimulus arranged to produce an H-reflex with the same latency as the MEP $(t=4 \mathrm{~ms})$; (4) FCR H-reflex conditioned by PPC CS using an ISI of $8 \mathrm{~ms}$, based on the relative latencies of FCR H-reflex and MEPs. In fact, these intervals were inappropriate for any median effects on the TMS pulse (Mariorenzi et al., 1991). Twenty responses were collected in each condition. Measurements were made in each individual trial on the peak-to peak amplitude of the $\mathrm{H}$ reflex or the MEP recorded from FCR.

Experiment 6: ipsilateral interactions in the left hemisphere. Ten subjects participated in this experiment. To stimulate the left M1, the intensity of TS was adjusted to evoke an MEP of $\sim 1 \mathrm{mV}$ peak to peak in the relaxed right FDI. The CS coil position for left PPC TMS was then defined relative to the $\mathrm{P} 3$ position of the 10-20 EEG system. The coil was positioned as in experiment 1 . The CS stimulus intensity was adjusted to be either suprathreshold (110\% RMT) or subthreshold (90\% RMT). ISIs between CS and TS were $4,6,8,10$, and 15 ms. In each block, we varied the intensity of the CS and the order of presentation of blocks varied pseudorandomly across subjects. Each block consisted of eighty trials. Seven conditions were randomly intermingled: TS alone (MEP) and CS1 plus TS (conditioned MEP for each seven different ISIs). Twenty responses were collected for test stimulus alone and 10 responses for conditioned MEP for each ISI. Measurements were made on each individual trial and the mean peak-to-peak amplitude of the conditioned MEP was expressed as a percentage of the mean peak-to-peak amplitude size of the unconditioned test pulse.

Data analysis. In experiments 1 and 6, the effects of PPC CS on the amplitude of MEPs recorded from stimulation of ipsilateral M1 were analyzed with separate ANOVAs for each intensity of stimulation with "condition" (TS alone, CS plus TS at various ISIs) as the main factor. We also performed two-way ANOVAs on the same data with ISI and "intensity" using normalized values calculated as the percentage of the mean peak-to-peak amplitude size of the unconditioned test pulse. In experiment 2, a two-way ANOVA on normalized values was performed with "coil current" and ISI as main factors. In experiment 3, a one-way ANOVA was performed on normalized values with ISI as the main factor, and a two-way ANOVA was performed on normalized values with "scalp site" and ISI as main factors. In experiment 4, two-way ANOVA was performed on mean of total counts for each PSTH period lasting $1.5 \mathrm{~ms}$ with condition (TS alone, CS plus TS at $4 \mathrm{~ms}$ ISI) and PSTH period as main factors. In experiment 5, paired $t$ tests were conducted to compare the effect of PPC CS on unconditioned H-reflex amplitude and unconditioned FCR MEP. A $p$ value $<0.05$ was considered significant. A significant main effect in the ANOVA was followed by post hoc paired $t$ test analysis with Bonferroni correction. The Greenhouse-Geisser correction was used for nonspherical data. Mauchley's test examined for sphericity.

\section{Results \\ Experiment 1}

Mean stimulation intensities for stimulation of the right PPC, measured as RMT over the ipsilateral M1, were $41.2 \%$ of the maximal stimulator output (range, 33-51\%). The major finding in experiment 1 was that a conditioning stimulus over the right PPC potentiated the ipsilateral motor cortex. The effect depended on the interstimulus interval and the conditioning intensity. In brief, MEPs were facilitated by PPC at ISIs of 4 and $15 \mathrm{~ms}$ when the CS intensity was 90\% RMT, but not at higher or lower intensities.

This was borne out in the ANOVA, in which a two-factor analysis of the whole data set showed significant main effects of intensity $(F=12.03 ; p<0.0005)$ and ISI $(F=3.21 ; p<0.05)$, although the interaction was not significant (Fig. $1 B$ ). Subsequent one-factor ANOVAs on the ISI data for each CS intensity showed main effects of ISI only when the CS intensity was $90 \%$ $(F=9,22 ; p<0.001)$, with post hoc $t$ tests comparing the amplitude of the conditioned and test MEP being significant only for 


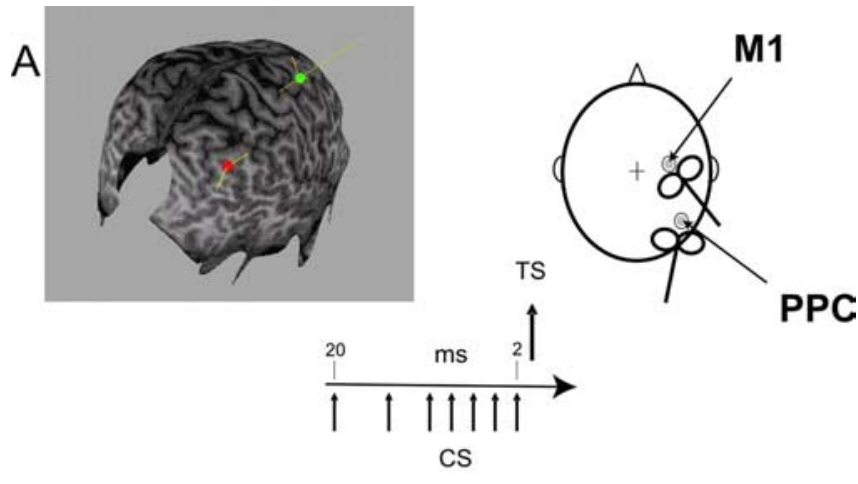

B
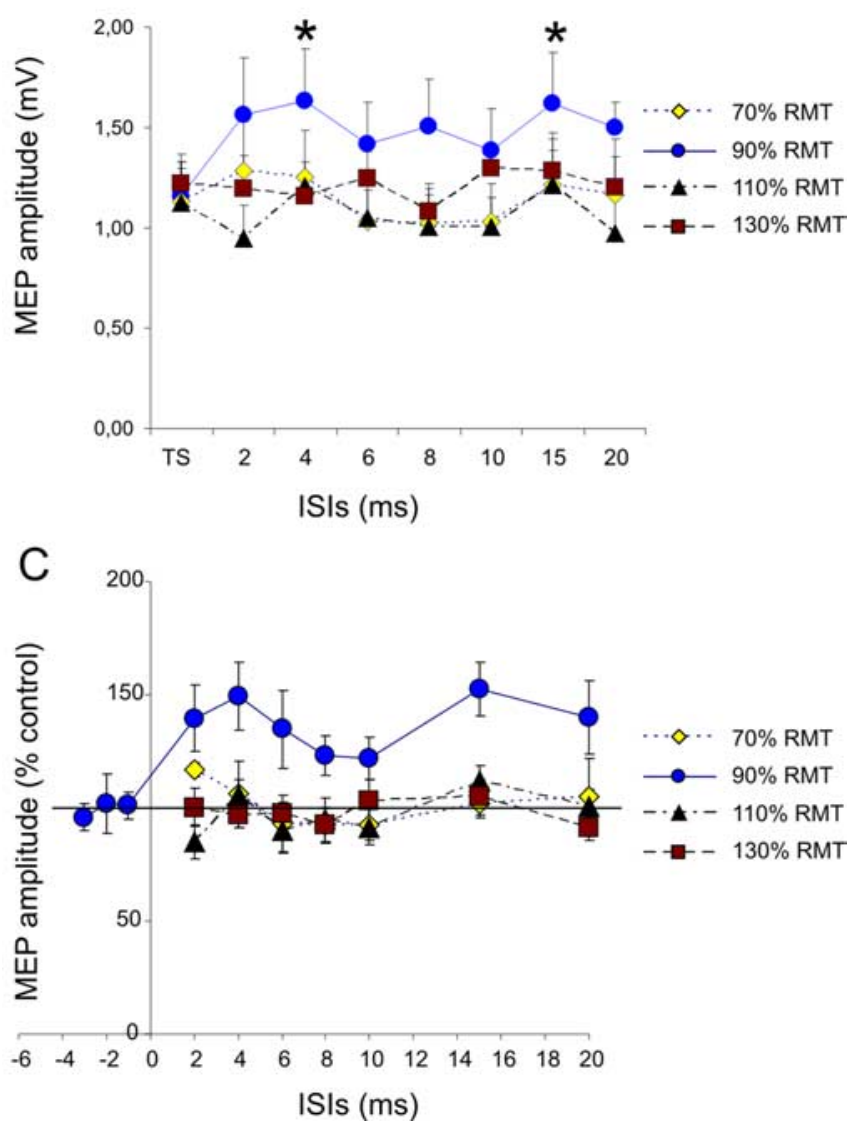

Figure 1. Effects of CS applied over the right PPC at different intensities on MEPs obtained by right M1 stimulation with subjects at rest. CS preceded TS applied over M1 by different ISIs ranging from 20 to $2 \mathrm{~ms}$. The relative orientations of the coils are shown. In each case, the monophasic current pulse flows into the handle of the coil, inducing current in the opposite direction in the underlying cortex. In the MRI reconstruction, the most anterior point (green dot) is at the junction with the postcentral sulcus (hand area of the motor cortex), and the posterior point (red dot) lies over the angular gyrus. The yellow lines represent the ideal trajectories of the magnetic fields; these lines terminate at the presumed site of stimulation. $\boldsymbol{A}$, Right PPC conditioning exerted potentiation over ipsilateral motor cortex. A single CS applied over the right PPC changed the amplitude of MEP obtained for ipsilateral M1 stimulation selectively when intensity of CS was set at $90 \%$ RMT with significant peaks obtained for ISIs of 4 and $15 \mathrm{~ms}$. B, № significant change was observed for lower (70\% RMT) or higher intensities of CS (110\%, 130\% RMT). Absolute values of MEPs in different conditions (mV) are shown in $\boldsymbol{B}$, whereas data in $\boldsymbol{C}$ are normalized and expressed as percentages of control test conditions. Data obtained in experiment 3, when TS preceded by 1,2, and 3 ms the CS set at $90 \%$ RMT intensity, are included in C. Errors bars indicate 1 SEM. Asterisks indicate a $p$ value $<0.05$ at post hoc analysis.
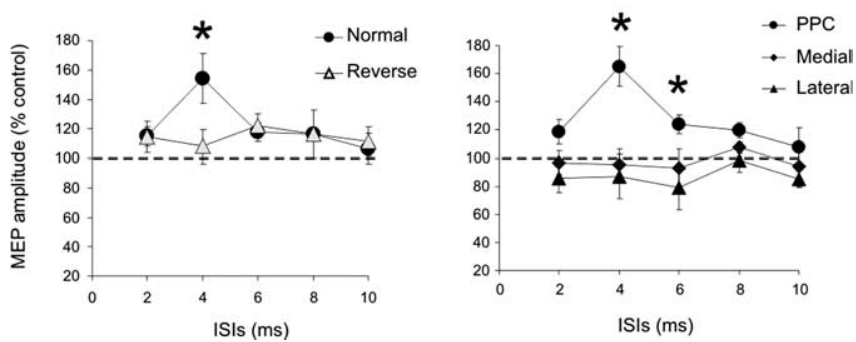

Figure 2. The effect of PPC conditioning is sensitive to the direction of current circulating in the coil. $\boldsymbol{A}$, In fact, potentiation of MEPs was obtained at $90 \%$ RMT only when the direction of the coil was the same as in experiment 1 but not when it was delivered with the opposite direction. $B$, There was facilitation selectively when $C S$ was applied over P4 but not $2 \mathrm{~cm}$ medially or laterally. Errors bars indicate 1 SEM. Asterisks indicate a $p$ value $<0.05$ at post hoc analysis.

CS intensity 90\% RMT at ISIs of 4 and $15 \mathrm{~ms}$ ( $p<0.01$ for both comparisons).

Figure $1 C$ plots the same data normalized to the amplitude of the test MEP (100\%), with the addition of the data at negative ISIs from the four subjects studied in this way. It is clear that although there is a weak tendency for an effect of ISI at other intensities, only CS at 90\% RMT show clear facilitation of MEPs evoked from M1. Furthermore, this finding was easily replicated in five subjects in which the experiment was performed in two different sessions. In those subjects, for the 4 ms peak of MEPs potentiation with CS set at 90\%, RMT intrinsic test-retest variability was minimal (mean coefficient of variation, $0.12 \pm 0.06$ ).

\section{Experiment 2}

Experiment 2 (Fig. $2 A$ ) showed that the effect of PPC stimulation is sensitive to the direction of current induced by the conditioning stimulus. The potentiation obtained at 90\% RMT using the orientation in experiment 1 was not evident when the CS was in the opposite direction (ANOVA direction $\times$ ISI interaction; $F=$ 3.34; $p<0.05)$. Post hoc analysis showed a significant difference only for 4 ms ISI $(p<0.01)$ but no other ISIs.

\section{Experiment 3}

Facilitation with a CS intensity of 90\% RMT was observed for stimulation over P4, but not $2 \mathrm{~cm}$ medially or laterally (ANOVA on data in Fig. $2 B$, site $\times$ ISIs interaction: $F=6.87 ; p<0.01)$. Post $h o c$ analysis showed that significant differences in the amount of facilitation were found for stimulation over $\mathrm{P} 4$ compared with the medial site at $4 \mathrm{~ms}(p<0.001)$ and $6 \mathrm{~ms}(p<0.01)$ and compared with the lateral site at $4 \mathrm{~ms}(p<0.001), 6 \mathrm{~ms}(p<$ $0.001)$, and $8 \mathrm{~ms}(p<0.05)$ (Figs. $2 B, 3 B)$.

Furthermore, when CS at $90 \%$ RMT and at 4 ms ISI was applied over different positions of the IPS, we found that potentiation of ipsilateral M1 occurred selectively for cIPS but not for mIPS or aIPS stimulation (ANOVA on data in Fig. $3 B$, site as the main factor: $F=3.57 ; p<0.05$ ). In fact, CS given over the anterior IPS actually suppressed MEPs at an ISI of $4 \mathrm{~ms}$, suggesting that this would be another potential site to examine input to M1 in future studies. However, this was not investigated in additional detail in the present study.

\section{Experiment 4}

Close inspection of the surface EMG responses in several subjects showed that potentiation of the MEP did not occur in the first few milliseconds of the response but was often delayed by $\sim 3 \mathrm{~ms}$ after 
A

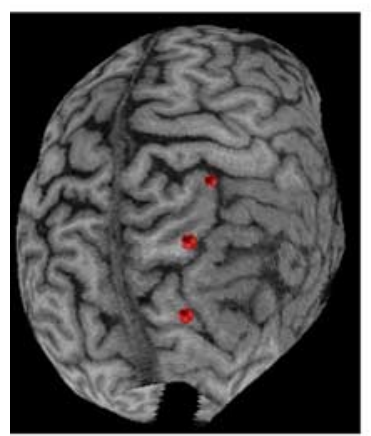

B

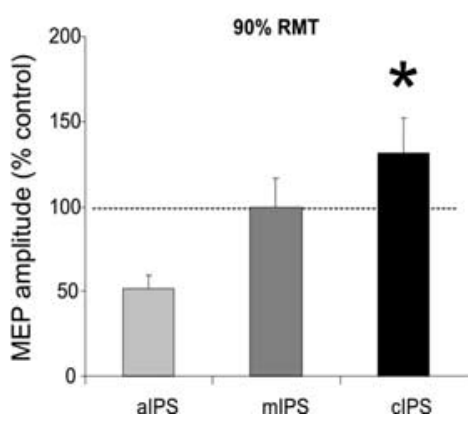

Figure 3. A, A three-dimensional structural MRI of one representative subject. The red dots represent the three cortical sites for the conditioning stimulus: top dot, alPS; middle dot, mIPS; bottom dot, cIPS. B, Facilitation of ipsilateral motor cortex was found selectively for stimulation of the cIPS. Errors bars indicate 1 SEM. Asterisks indicate a $p$ value $<0.05$ at post hoc analysis.

onset. This is illustrated in the superimposition of test and conditioned MEPs at an ISI of $4 \mathrm{~ms}$ from one subject in Figure $4 \mathrm{~A}$.

We analyzed this delay in onset of facilitation in more detail by performing PSTH analysis of the firing pattern of SMU recorded via needle EMG. Figure $4 B$ shows the combined PSTH data from 10 SMU. In Figure 4, $C$ and $D$, the same data has been aligned to the onset of the response to the test stimulus alone (the $\mathrm{D}$ wave at $0 \mathrm{~ms}$ ). The counts were then grouped into three time bins corresponding to the three main peaks in the PSTH (see Materials and Methods), and a repeated-measures ANOVA performed on the data from the 10 SMU with condition (M1 alone or PPC-M1) and peak as main factors. This revealed a significant condition $X$ peak interaction $(F=5.16 ; p<0.01)$, demonstrating that PPC CS modified the time course of the PSTHs. Post hoc analysis showed that PPC conditioning at an ISI of $4 \mathrm{~ms}$ led to significantly more counts in the late peak $(6.67 \pm 1.52$ vs $11.11 \pm 2.19 ; p<0.001)$ compared with unconditioned M1 stimulation.

\section{Experiment 5}

PPC CS did not change the amplitude of the $\mathrm{H}$ reflex recorded from FCR $(0.74 \pm 0.34$ vs $0.76 \pm 0.32 \mathrm{mV} ; t=0.39)$, although it significantly increased the amplitude of the FCR MEP (0.63 \pm 0.18 vs $0.78 \pm 0.22 \mathrm{mV} ; t=0.04$ ) (Fig. 5 ).

\section{Experiment 6}

In this experiment (Fig. 6), we examined the effect of giving the CS over the left PPC on the MEP evoked by a TS over the left M1. As in the right hemisphere, we found that a CS over the left PPC significantly potentiated the left motor cortex, although with subtle differences in timing. As with the right hemisphere, CS over the left PPC potentiated the left M1 when the intensity was 90\% RMT (ANOVA with condition main factor: $F=8.75 ; p<$ 0.01 ) but not at $110 \%$ RMT. Post hoc analysis for $90 \%$ RMT showed that potentiation was obtained at ISIs of $4 \mathrm{~ms}(1.15 \pm$ $0.45 \mathrm{mV}$ vs $1.58 \pm 0.71 \mathrm{mV} ; p<0.01)$ and $6 \mathrm{~ms}(1.15 \mathrm{vs} 1.62 \pm$ $0.66 \mathrm{mV} ; p<0.01)$. Direct comparison of the two conditioning intensities in each subject was made in a separate analysis using normalized values (test MEP alone $=100 \%$ ). Two-way ANOVA showed a significant main effect of intensity $(F=6.72 ; p<0.001)$ and a significant intensity $\times$ ISI interaction $(F=5.24 ; p<0.001)$ with post hoc analysis showing significant differences selectively at an ISI of $4 \mathrm{~ms}(128.2 \mathrm{vs} 105.9 \% ; p<0.05)$ and at an ISI of $6 \mathrm{~ms}$ (136.2 vs $93.5 \% ; p<0.0001$ ) (Fig. 6).
This was confirmed by subsequent one-factor ANOVAs on the ISI data for each CS intensity that showed main effects of ISI only when the CS intensity was $90 \%(F=6.45 ; p<0.01)$, with post hoc $t$ tests comparing the amplitude of the conditioned and test MEP being significant only for CS intensity $90 \%$ RMT at an ISI of 4 and $6 \mathrm{~ms}$ ( $p<0.05$ for both comparisons).

\section{Discussion}

The present data show that in subjects at rest, conditioning stimuli over a specific site in the right posterior parietal cortex potentiates MEPs evoked from ipsilateral M1 if the intensity of the CS is $90 \%$ RMT and the ISI is $\sim 4$ or $15 \mathrm{~ms}$. Similar results were obtained in the left hemisphere with a slightly different time course. We argue below that these effects occur because of corticocortical projections from the PPC to frontal areas of the cortex via the superior longitudinal fasiculus. If so, this may be a useful method of testing the excitability of these connections during different types of movement as well as in patients with neurological diseases.

\section{Mechanisms for ipsilateral facilitation}

Several recent studies that have used the same paired TMS method as in the present study have focused on connections between motor and premotor areas (Civardi et al., 2001; Mochizuki et al., 2004; Shimazu et al., 2004; Baumer et al., 2006; Koch et al., 2006, 2007). As in the present study, the effects depended on both ISI as well as the intensity of the CS, and in all cases, the interactions were thought to depend on corticocortical projections between PMd and M1 (Marconi et al., 2003; Dum and Strick, 2005). Indeed, a recent investigation in monkeys demonstrated that electrical stimulation of ventral premotor (PMd was not tested) led $3 \mathrm{~ms}$ later to facilitation of late I-wave interneurons in M1 (Shimazu et al., 2004), providing physiological evidence for direct corticocortical projections between these areas.

The initial potentiation that we observed after stimulation over the right PPC peaked at an ISI of $4 \mathrm{~ms}$ and was evoked with a CS intensity of $90 \%$ RMT. By analogy with known effects on M1 (Chen et al., 2003), a CS of this intensity would be able to activate outputs from the site of stimulation. Because there are no direct projections to the spinal cord from the PPC, and we did not observe any change on the excitability of spinal $\mathrm{H}$-reflex induced by PPC conditioning, we assume that the interaction with the effect of the M1 test pulse resulted from activation of corticocortical pathways. Although an ISI of $4 \mathrm{~ms}$ appears very short for transmission from PPC to M1, estimation of the true latency of the interaction is complicated by the fact that the TS to M1 evokes a series of I-wave volleys in corticospinal neurons that can last several milliseconds. These then summate at the spinal cord to produce the MEP in muscle. To obtain more information about the early facilitation at an ISI of $4 \mathrm{~ms}$, we therefore performed single motor unit studies to determine which components of the descending volley were affected by potentiation from the PPC.

We found that PPC conditioning increased the relative amplitude of late motor unit facilitation compared with unconditioned M1 stimulation. Indeed, in some subjects, this was observable even in MEPs recorded with surface electrodes in which facilitation of MEPs appeared to start $3 \mathrm{~ms}$ or more after onset of the response at a time when late I-wave inputs would contribute to motor unit activation. We hypothesize that PPC input to M1 preferentially targets late I-wave input to corticospinal neurons. These units are first recruited and may only contribute a little to the surface potentials obtained with a larger test pulse in the main experiment. We assume that the larger SMUs that are recruited 
with larger test pulses behave in the same way as the small ones we studied, and therefore, the potentiation of MEPs induced by PPC conditioning could also be explained in terms of corticocortical pathways that interact with the late I-wave circuits. Future studies are needed to address whether PPC conditioning might affect other interneuronal circuits in the motor cortex, such as those involved in the inhibitory circuits, like the silent period (Rossini et al., 1995).

The main fiber tract connecting the PPC with frontal areas is the SLF (Dejerine, 1895). Recently, the four subdivisions of the SLF previously described in nonhuman primates have been identified and segmented in humans using diffusion tensor MRI (Makris et al., 2005). This showed that the SLF I is situated within the white matter of the superior parietal lobule (BA 7) and the post central and precentral gyrus (areas 4 and 5). The SLF II is located within the white matter of the angular gyrus (areas 39 and 40), postcentral gyrus (areas 3, 1, and 2), precentral gyrus (area 4 ), and middle frontal gyrus (areas 6 and 46). SFL III is situated more laterally in the white matter of the parietal and frontal opercula, and the fourth subdivision of the SLF is the arcuate fascicule extending from the superior temporal gyrus to the lateral prefrontal cortex. However, because of the methodology adopted (diffusion tensor-MRI), they could not investigate where the fibers actually originate and terminate.

In our study, the center of the conditioning coil was positioned over a scalp site corresponding to a part of the angular gyrus in the IPL and close to cIPS. Therefore, we stimulated a cortical region where there is a reasonable anatomical assumption that connectivity to the motor cortex may be present (Grefkes and Fink, 2005). To the best of our knowledge, this is the first demonstration in humans that it may exist functionally and that activation of some of these fibers may result in facilitation of the motor cortex. Our findings using neuronavigation in three subjects suggest that the early peak of facilitation (4-6 ms) may depend on activation of direct corticocortical projections originating from cIPS/IPL or from the underlying white matter (i.e., $\mathrm{SLF}$ ) that terminate in M1. However, further imaging work is needed to confirm this on more subjects in the future. Indeed, more investigations are needed to disclose whether stimulation of the PPC activates the output axons via cortical interneurons or directly goes through fibers at the axonal hillock level. Furthermore, we cannot exclude a contribution from activity in polysynaptic circuits linking the PPC and premotor cortex with secondary connections in M1. In fact, a consistent body of animal literature indicates that the majority of corticocortical projections originating in PPC reach the premotor cortex, with only a minority terminating directly on the primary motor cortex (Petrides and Pandya, 1988; Picard and Strick, 1996; Matelli et al., 1998; Battaglia-Mayer et al., 2003). It may be that the late facilitation observed at $15 \mathrm{~ms}$ ISI in the right hemisphere is because of activity in polysynaptic circuits involving premotor areas. Additional investigations with behavioral tasks sensitive to the premotor cortex activity could add useful information in future studies, as would combined techniques such as TMS/EEG (Bonato et al.,
B
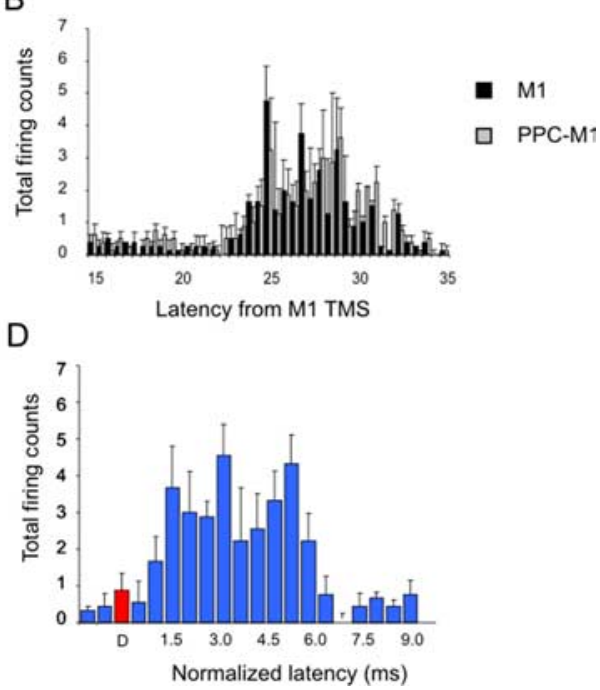

Figure 4. $P P C$ conditioning exerts facilitation of ipsilateral $M 1$ trough corticocortical projections that interact with the late components of MEPs. A, Average surface MEPs from a representative subject for TS (red line) and CS (90\% RMT with 4 ms ISI, gray preceded by PPC CS (gray bars). PPC conditioning significantly modified the firing probability of PSTHs components. C, D, Com-

A

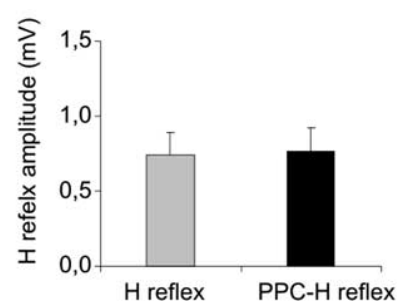

B

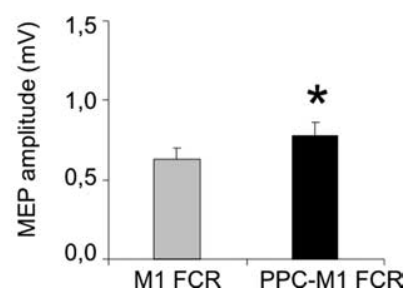

Figure 5. Effects of $P P C$ conditioning on H-reflexes and MEPs recorded from the FCR muscle. Average data from five subjects. $A$, No significant modulation of $H$-reflex was found during PPC conditioning. B, FCR MEP amplitude was potentiated during PPC conditioning ( $90 \%$ intensity; $\mid \mathrm{ISI}, 4 \mathrm{~ms})$. Error bars indicate $1 \mathrm{SEM}$. Asterisks indicate a $p$ value $<0.05$ at post hoc analysis.

2006) that could provide direct mapping of such hypothesized connections.

\section{Facilitation versus inhibition in parietomotor connections}

Previous studies using paired-pulse TMS to explore connections to the motor cortex from the cerebellum, PMd, and contralateral M1, have described a mixture of excitatory and inhibitory effects with the latter usually being the most prominent (Ferbert et al., 1992; Ugawa et al., 1995; Civardi et al., 2001; Mochizuki et al., 2004; Baumer et al., 2006; Koch et al., 2006, 2007). Indeed, in the case of inputs from contralateral M1 or PMd, facilitation is only revealed with very low intensity conditioning stimuli and short ISIs, whereas at higher intensities and longer intervals, the effect is swamped by inhibition. In such cases, it is assumed that facilitatory and inhibitory effects are mediated by neurons that have different thresholds and that the lowest threshold group (in these cases facilitatory) can be selectively recruited by adjusting the stimulus intensity. It is less clear why inhibition is so prominent. One possibility (Hanajima et al., 2001) is that many connections are organized in a center-surround pattern of point-to point facilitation and widespread inhibition. The relatively low focality of 


\section{A}

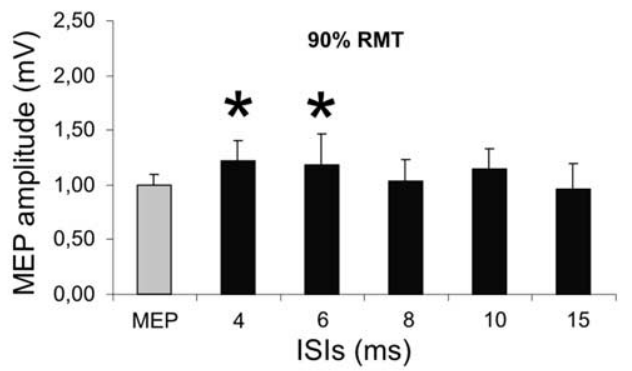

B

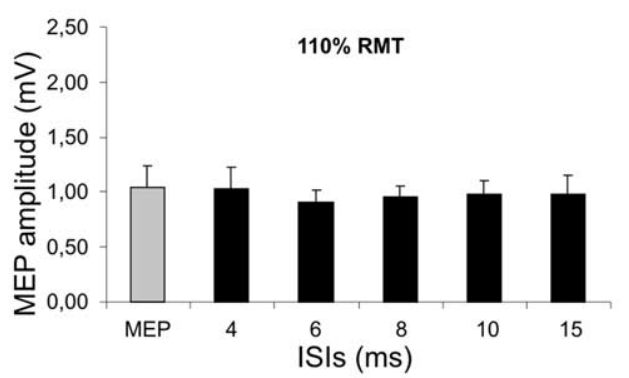

C

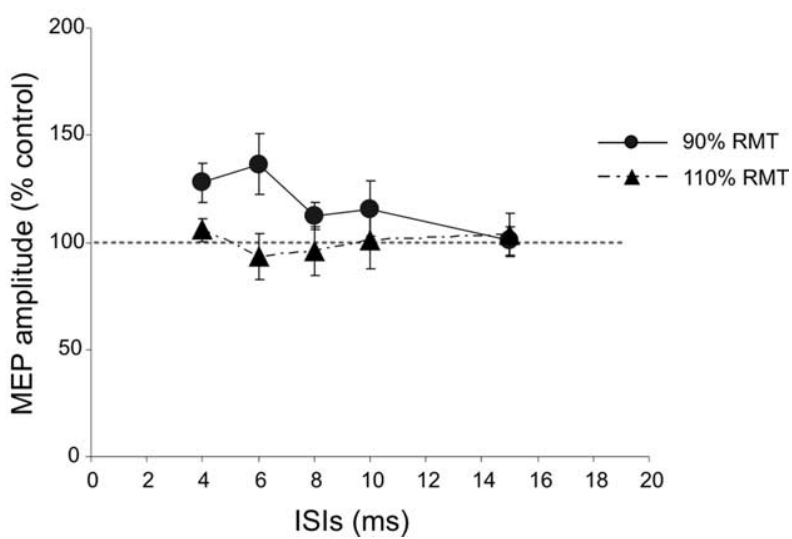

Figure 6. Effects of CS applied over left PPC at different intensities on MEPs obtained by left M1 stimulation. CS preceded TS applied over M1 by different ISIs ranging from 15 to $4 \mathrm{~ms}$. A, A single CS applied over the left PPC potentiated the amplitude of MEP obtained for ipsilateral M1 stimulation selectively when intensity of $C S$ was set at $90 \%$ RMT with significant peaks obtained for ISIs of 4 and 6 ms. B, No significant change was observed for higher intensities of CS (110\% RMT). Data in ( are normalized and expressed as a percentage of control test condition. Errors bars indicate $1 \mathrm{SEM}$. Asterisks indicate a $p$ value $<0.05$ at post hoc analysis.

TMS may favor recruitment of the inhibitory surround and mask point-to-point facilitation. In the present study, we found no evidence for inhibition from PPC to M1 despite exploring a variety of ISIs and stimulus intensities. Inhibition did occur, however, from the most anterior IPS site that we examined, but this was not studied in detail. We conclude that the organization of inputs from the PPC differs from that from the premotor and contralateral motor cortex. This may be, for example, because facilitation and inhibition arise from different locations and have different recruitment threshold to TMS, but additional work is needed before any firm conclusions can be made.

\section{Conclusions}

The present results demonstrate the existence of facilitatory parietomotor connections that may be activated by means of TMS. Testing how this is impaired in different neurological disorders could lead to novel insights in the understanding of the pathophysiology of various neuropsychiatric disorders. Furthermore applications in pathological conditions, such as patients with focal lesion of the PPC, could provide support to the hypothesis that the interaction observed in the present study depends on activation of specific corticocortical projections. We note, however, that the noise and scalp tingling on the healthy and affected hemisphere in neglect patients would require careful control experiments to eliminate any placebo effects. Finally, previous studies in nonhuman primates have shown that a large component of PPC activity seems to be correlated with movement planning (Cohen and Andersen, 2002). Indeed, PPC receives inputs in many different modalities (visual, auditory, and somatosensory), and it has been shown that regions of the PPC are involved in planning of movements to targets of different sensory modalities (Cavada and Goldman-Rakic, 1989; Seltzer and Pandya, 1994; Johnson et al., 1996; Shadlen and Newsome, 1996, 2001; Linden et al., 1999; Snyder et al., 2000; Quian Quiroga et al., 2006). We therefore speculate that the connection we have studied in the present work may be important in movement planning in humans.

\section{References}

Battaglia-Mayer A, Caminiti R, Lacquaniti F, Zago M (2003) Multiple levels of representation of reaching in the parieto-frontal network. Cereb Cortex 13:1009-1022.

Baumer T, Bock F, Koch G, Lange R, Rothwell JC, Siebner HR, Munchau A (2006) Magnetic stimulation of human premotor or motor cortex produces interhemispheric facilitation through distinct pathways. J Physiol (Lond) 572:857-868.

Bestmann S, Baudewig J, Siebner HR, Rothwell JC, Frahm J (2004) Functional MRI of the immediate impact of transcranial magnetic stimulation on cortical and subcortical motor circuits. Eur J Neurosci 19:1950-1962.

Bestmann S, Baudewig J, Siebner HR, Rothwell JC, Frahm J (2005) BOLD MRI responses to repetitive TMS over human dorsal premotor cortex. NeuroImage 28:22-29.

Bonato C, Miniussi C, Rossini PM (2006) Transcranial magnetic stimulation and cortical evoked potentials: a TMS/EEG co-registration study. Clin Neurophysiol 117:1699-1707.

Cavada C, Goldman-Rakic PS (1989) Posterior parietal cortex in rhesus monkey: I. Parcellation of areas based on distinctive limbic and sensory corticocortical connections. J Comp Neurol 287:393-421.

Chen R, Yung D, Li JY (2003) Organization of ipsilateral excitatory and inhibitory pathways in the human motor cortex. J Neurophysiol 89:1256-1264.

Civardi C, Cantello R, Asselman P, Rothwell JC (2001) Transcranial magnetic stimulation can be used to test connections to primary motor areas from frontal and medial cortex in humans. NeuroImage 14:1444-1453.

Cohen YE, Andersen RA (2002) A common reference frame for movement plans in the posterior parietal cortex. Nat Rev Neurosci 3:553-562.

Croxson PL, Johansen-Berg H, Behrens TE, Robson MD, Pinsk MA, Gross CG, Richter W, Richter MC, Kastner S, Rushworth MF (2005) Quantitative investigation of connections of the prefrontal cortex in the human and macaque using probabilistic diffusion tractography. J Neurosci 25:8854-8866.

Dejerine J (1895) Anatomie des centres nerveux. Paris: Reuff et Cie.

Dum RP, Strick PL (2005) Frontal lobe inputs to the digit representations of the motor areas on the lateral surface of the hemisphere. J Neurosci 25:1375-1386.

Ferbert A, Priori A, Rothwell JC, Day BL, Colebatch JG, Marsden CD (1992) Interhemispheric inhibition of the human motor cortex. J Physiol (Lond) 453:525-546.

Grefkes C, Fink GR (2005) The functional organization of the intraparietal sulcus in humans and monkeys. J Anat 207:3-17.

Hanajima R, Ugawa Y, Machii K, Mochizuki H, Terao Y, Enomoto H, Furubayashi T, Shiio Y, Uesugi H, Kanazawa I (2001) Interhemispheric facilitation of the hand motor area in humans. J Physiol (Lond) 531:849-859.

Hanajima R, Ugawa Y, Terao Y, Enomoto H, Shiio Y, Mochizuki H, Furuba- 
yashi T, Uesugi H, Iwata NK, Kanazawa I (2002) Mechanisms of intracortical I-wave facilitation elicited with paired-pulse magnetic stimulation in humans. J Physiol (Lond) 538:253-261.

Herwig U, Satrapi P, Schonfeldt-Lecuona C (2003) Using the international 10-20 EEG system for positioning of transcranial magnetic stimulation. Brain Topogr 16:95-99.

Hortobágyi T, Taylor JL, Petersen NT, Russell G, Gandevia SC (2003) Changes in segmental and motor cortical output with contralateral muscle contractions and altered sensory inputs in humans. J Neurophysiol 90:2451-2459.

Johnson PB, Ferraina S, Bianchi L, Caminiti R (1996) Cortical networks for visual reaching: physiological and anatomical organization of frontal and parietal lobe arm regions. Cereb Cortex 6:102-119.

Koch G, Franca M, Fernandez Del Olmo M, Cheeran B, Milton R, Alvarez Sauco M, Rothwell JC (2006) Time course of functional connectivity between dorsal premotor and contralateral motor cortex during movement selection. J Neurosci 26:7452-7459.

Koch G, Franca M, Mochizuki H, Marconi B, Caltagirone C, Rothwell JC (2007) Interactions between pairs of transcranial magnetic stimuli over the human left dorsal premotor cortex differ from those seen in primary motor cortex. J Physiol (Lond) 578:551-562.

Lee L, Siebner HR, Rowe JB, Rizzo V, Rothwell JC, Frackowiak RS, Friston KJ (2003) Acute remapping within the motor system induced by lowfrequency repetitive transcranial magnetic stimulation. J Neurosci 23:5308-5318.

Linden JF, Grunewald A, Andersen RA (1999) Responses to auditory stimuli in macaque lateral intraparietal area. II. Behavioral modulation. J Neurophysiol 82:343-358.

Makris N, Kennedy DN, McInerney S, Sorensen AG, Wang R, Caviness Jr VS, Pandya DN (2005) Segmentation of subcomponents within the superior longitudinal fascicle in humans: a quantitative, in vivo, DT-MRI study. Cereb Cortex 15:854-869.

Marconi B, Genovesio A, Giannetti S, Molinari M, Caminiti R (2003) Callosal connections of dorso-lateral premotor cortex. Eur J Neurosci 18:775-788.

Mariorenzi R, Zarola F, Caramia MD, Paradiso C, Rossini PM (1991) Noninvasive evaluation of central motor tract excitability changes following peripheral nerve stimulation in healthy humans. Electroencephalogr Clin Neurophysiol 81:90-101.

Matelli M, Govoni P, Galletti C, Kutz DF, Luppino G (1998) Superior area 6 afferents from the superior parietal lobule in the macaque monkey. J Comp Neurol 402:327-352.

Meynert (1865) Anatomie der hirnrinde und ihre verbindungsbahnen mit den empfindenden oberflachen und den bewegenden massen. In: Leidesdorf's lehrbuch der phychischen krankheiten. Erlangen, Germany.

Mochizuki H, Huang YZ, Rothwell JC (2004) Interhemispheric interaction between human dorsal premotor and contralateral primary motor cortex. J Physiol (Lond) 561:331-338.

Mountcastle VB, Lynch JC, Georgopoulos A, Sakata H, Acuna C (1975) Posterior parietal association cortex of the monkey: command functions for operations within extrapersonal space. J Neurophysiol 38:871-908.

Murase N, Duque J, Mazzocchio R, Cohen LG (2004) Influence of interhemispheric interactions on motor function in chronic stroke. Ann Neurol 55:400-409.

Oliveri M, Rossini PM, Traversa R, Cicinelli P, Filippi MM, Pasqualetti P, Tomaiuolo F, Caltagirone C (1999) Left frontal transcranial magnetic stimulation reduces contralesional extinction in patients with unilateral right brain damage. Brain 122:1731-1739.

Oliveri M, Rossini PM, Filippi MM, Traversa R, Cicinelli P, Palmieri MG, Pasqualetti P, Caltagirone C (2000) Time-dependent activation of parieto-frontal networks for directing attention to tactile space. A study with paired transcranial magnetic stimulation pulses in right-braindamaged patients with extinction. Brain 123:1939-1947.

Paus T, Jech R, Thompson CJ, Comeau R, Peters T, Evans AC (1997) Transcranial magnetic stimulation during positron emission tomography: a new method for studying connectivity of the human cerebral cortex. J Neurosci 17:3178-3184.

Petrides M, Pandya DN (1988) Association fiber pathways to the frontal cortex from the superior temporal region in the rhesus monkey. J Comp Neurol 273:52-66.

Picard N, Strick PL (1996) Motor areas of the medial wall: a review of their location and functional activation. Cereb Cortex 6:342-353.

Quian Quiroga R, Snyder LH, Batista AP, Cui H, Andersen RA (2006) Movement intention is better predicted than attention in the posterior parietal cortex. J Neurosci 26:3615-3620.

Rossini PM, Barker AT, Berardelli A, Caramia MD, Caruso G, Cracco RQ, Dimitrijevic MR, Hallett M, Katayama Y, Lucking $\mathrm{CH}$, Maertens de Noordhout AL, Marsden CD, Murray NMF, Rothwell JC, Swash M, Tomberg C (1994) Non-invasive electrical and magnetic stimulation of the brain, spinal cord and roots: basic principles and procedures for routine clinical application. Report of an IFCN committee. Electroencephalogr Clin Neurophysiol 91:79-92.

Rossini PM, Caramia MD, Iani C, Desiato MT, Sciarretta G, Bernardi G (1995) Magnetic transcranial stimulation in healthy humans: influence on the behavior of upper limb motor units. Brain Res 676:314-324.

Rozzi S, Calzavara R, Belmalih A, Borra E, Gregoriou GG, Matelli M, Luppino G (2006) Cortical connections of the inferior parietal cortical convexity of the macaque monkey. Cereb Cortex 16:1389-1417.

Rushworth MF, Taylor PC (2006) TMS in the parietal cortex: updating representations for attention and action. Neuropsychologia 44:2700-2716.

Rushworth MF, Behrens TE, Johansen-Berg H (2006) Connection patterns distinguish 3 regions of human parietal cortex. Cereb Cortex 16: $1418-1430$

Seltzer B, Pandya DN (1994) Parietal, temporal, and occipital projections to cortex of the superior temporal sulcus in the rhesus monkey: a retrograde tracer study. J Comp Neurol 343:445-463.

Shadlen MN, Newsome WT (1996) Motion perception: seeing and deciding. Proc Natl Acad Sci USA 93:628-633.

Shadlen MN, Newsome WT (2001) Neural basis of a perceptual decision in the parietal cortex (area LIP) of the rhesus monkey. J Neurophysiol 86:1916-1936.

Shimazu H, Maier MA, Cerri G, Kirkwood PA, Lemon RN (2004) Macaque ventral premotor cortex exerts powerful facilitation of motor cortex outputs to upper limb motoneurons. J Neurosci 24:1200-1211.

Snyder LH, Batista AP, Andersen RA (2000) Intention-related activity in the posterior parietal cortex: a review. Vision Res 40:1433-1441.

Tanne-Gariepy J, Rouiller EM, Boussaoud D (2002) Parietal inputs to dorsal versus ventral premotor areas in the macaque monkey: evidence for largely segregated visuomotor pathways. Exp Brain Res 145:91-103.

Ugawa Y, Uesaka Y, Terao Y, Hanajima R, Kanazawa I (1995) Magnetic stimulation over the cerebellum in humans. Ann Neurol 37:703-713. 